\title{
Approximate Solutions for Local Fractional Linear Transport Equations Arising in Fractal Porous Media
}

\author{
Meng Li, ${ }^{1}$ Xiao-Feng Hui, ${ }^{1}$ Carlo Cattani, ${ }^{2}$ Xiao-Jun Yang, ${ }^{3}$ and Yang Zhao ${ }^{4,5}$ \\ ${ }^{1}$ School of Management, Harbin Institute of Technology, Harbin 150001, China \\ ${ }^{2}$ Department of Mathematics, University of Salerno, Via Ponte don Melillo, Fisciano, 84084 Salerno, Italy \\ ${ }^{3}$ Department of Mathematics and Mechanics, China University of Mining and Technology, Xuzhou, Jiangsu 221008, China \\ ${ }^{4}$ Electronic and Information Technology Department, Jiangmen Polytechnic, Jiangmen 529090, China \\ ${ }^{5}$ College of Instrumentation \& Electrical Engineering, Jilin University, Changchun 130061, China
}

Correspondence should be addressed to Yang Zhao; zhaoyang19781023@gmail.com

Received 2 March 2014; Accepted 25 March 2014; Published 14 April 2014

Academic Editor: Gongnan Xie

Copyright (c) 2014 Meng Li et al. This is an open access article distributed under the Creative Commons Attribution License, which permits unrestricted use, distribution, and reproduction in any medium, provided the original work is properly cited.

We investigate the local fractional linear transport equations arising in fractal porous media by using the local fractional variational iteration method. Their approximate solutions within the nondifferentiable functions are obtained and their graphs are also shown.

\section{Introduction}

Transport equations [1-3] had successful applications in aeronomy [4], semiconductors [5], superconductor [6], turbulence [7], QCD [8], plasma [9], gas mixture [10], and biology [11]. The linear transport equation was written as follows [12]:

$$
\frac{\partial u(x, t)}{\partial t}+a(x, t) \frac{\partial u(x, t)}{\partial x}=0
$$

subject to the initial condition

$$
u(x, 0)=f(x),
$$

where $f(x)$ is a continuous function (differentiable function).

With the development of the transport theory in porous media [13], the disordered materials, which show fractal characteristics [14], had been investigated [15-17]. Recently, during anomalous transport [18] and evidence of its existence in point vortex flow [19], fractional theory of transport was developed by many researchers. Zaslavsky [20] and Tarasov [21] investigated the anomalous transport of fractional dynamics. Lutz presented the transport equations for Lévy stable processes with fractional derivative [22]. Uchaikin and
Sibatov considered the applications in disordered semiconductors [23]. Metzler and Klafter reported developments in the description of anomalous transport based upon fractional derivative [24]. Kadem et al. studied the solutions for the fractional transport equation by using the spectral method [25].

The above results via fractional calculus are set upon the differentiable functions. However, there are many nondifferentiable functions, which do not deal with the classical and fractional calculus. More recently, the local fractional calculus developed in [26-36] was the best candidate for scientists to deal with the nondifferentiable functions. In this paper, we consider the local fractional linear transport equations arising in fractal porous media in one-dimensional case [28]:

$$
\frac{\partial^{\alpha} u(x, t)}{\partial t^{\alpha}}+a(x, t) \frac{\partial^{\alpha} u(x, t)}{\partial x^{\alpha}}=0
$$

subject to the initial condition

$$
u(x, 0)=f(x),
$$

where the velocity term $a(x, t)$ and the quantity $f(x)$ may be nondifferentiable functions. The purpose of the current 
paper is to find the nondifferentiable solutions for the local fractional linear transport equations arising in fractal porous media in one-dimensional case by using the local fractional variational iteration method [31-36]. The plan of the paper is as follows. In Section 2, the conceptions of local fractional derivatives and local fractional integrals are given. In Section 3, the idea of local fractional variational iteration method is presented. In Section 4, the nondifferentiable behaviors for solutions of local fractional linear transport equations are studied. In Section 5, the conclusions are given.

\section{On the Local Fractional Calculus}

In this section, we introduce the definitions of local fractional derivatives and integrals which are used in the paper.

We set the function $[26,27]$

$$
f(x) \in C_{\alpha}(a, b)
$$

where

$$
\left|f(x)-f\left(x_{0}\right)\right|<\varepsilon^{\alpha},
$$

with $\left|x-x_{0}\right|<\delta$, for $\varepsilon>0,0<\alpha<1$ and $\varepsilon \in R$.

Definition 1. Let $f(x) \in C_{\alpha}(a, b)$. We define the local fractional derivative of $f(x)$ of order $\alpha$ by [26-36]

$$
\frac{d^{\alpha} f\left(x_{0}\right)}{d x^{\alpha}}=\frac{\Delta^{\alpha}\left(f(x)-f\left(x_{0}\right)\right)}{\left(x-x_{0}\right)^{\alpha}},
$$

where

$$
\Delta^{\alpha}\left(f(x)-f\left(x_{0}\right)\right) \cong \Gamma(1+\alpha)\left[f(x)-f\left(x_{0}\right)\right] .
$$

Definition 2. Let $f(x) \in C_{\alpha}[a, b]$. We define the local fractional integral of $f(x)$ of order $\alpha$ in the interval $[a, b]$ by [26, 27, 29-36]

$$
\begin{aligned}
{ }_{a} I_{b}^{(\alpha)} f(x) & =\frac{1}{\Gamma(1+\alpha)} \int_{a}^{b} f(t)(d t)^{\alpha} \\
& =\frac{1}{\Gamma(1+\alpha)} \lim _{\Delta t \rightarrow 0} \sum_{j=0}^{j=N-1} f\left(t_{j}\right)\left(\Delta t_{j}\right)^{\alpha},
\end{aligned}
$$

where the partitions of the interval $[a, b]$ are denoted as $\left(t_{j}, t_{j+1}\right), j=0, \ldots, N-1, t_{0}=a$ and $t_{N}=b$ with $\Delta t_{j}=t_{j+1}-t_{j}$ and $\Delta t=\max \left\{\Delta t_{0}, \Delta t_{1}, \Delta t_{j}, \ldots\right\}$.

From (8) and (9), there are some properties in the following form [26, 27, 31-35]:

$$
\begin{aligned}
\frac{d^{\alpha}}{d x^{\alpha}} \frac{x^{n \alpha}}{\Gamma(1+n \alpha)} & =\frac{x^{(n-1) \alpha}}{\Gamma(1+(n-1) \alpha)}, \\
\frac{d^{\alpha}}{d x^{\alpha}} E_{\alpha}\left(x^{\alpha}\right) & =E_{\alpha}\left(x^{\alpha}\right),
\end{aligned}
$$

$$
\begin{gathered}
\frac{d^{\alpha}}{d x^{\alpha}} \sin _{\alpha}\left(x^{\alpha}\right)=\cos _{\alpha}\left(x^{\alpha}\right), \\
\frac{d^{\alpha}}{d x^{\alpha}} \sinh _{\alpha}\left(x^{\alpha}\right)=\cosh _{\alpha}\left(x^{\alpha}\right), \\
{ }_{0} I_{x}^{(\alpha)} \frac{x^{n \alpha}}{\Gamma(1+n \alpha)}=\frac{x^{(n+1) \alpha}}{\Gamma(1+(n+1) \alpha)},
\end{gathered}
$$

where

$$
\begin{gathered}
E_{\alpha}\left(x^{\alpha}\right)=\sum_{k=0}^{\infty} \frac{x^{\alpha k}}{\Gamma(1+k \alpha)}, \\
\sin _{\alpha}\left(x^{\alpha}\right)=\sum_{k=0}^{\infty}(-1)^{k} \frac{x^{(2 k+1) \alpha}}{\Gamma[1+(2 k+1) \alpha]} \\
\cos _{\alpha}\left(x^{\alpha}\right)=\sum_{k=0}^{\infty}(-1)^{k} \frac{x^{2 \alpha k}}{\Gamma(1+2 \alpha k)} \\
\sinh _{\alpha}\left(x^{\alpha}\right)=\sum_{k=0}^{\infty} \frac{x^{(2 k+1) \alpha}}{\Gamma[1+(2 k+1) \alpha]} \\
\cosh _{\alpha}\left(x^{\alpha}\right)=\sum_{k=0}^{\infty} \frac{x^{2 \alpha k}}{\Gamma(1+2 \alpha k)} .
\end{gathered}
$$

\section{The Local Fractional Variational Iteration Method}

In this section, the local fractional variational iteration method first proposed in [31] is applied to deal with the local fractional linear differential equations of order $\alpha$.

Let us consider the following local fractional operator equation:

$$
L_{\alpha} u(s)+N_{\alpha} u(s)=0,
$$

where the linear local fractional differential operator is defined as $L_{\alpha}=d^{\alpha} / d s^{\alpha}$, a nonlinear local fractional operator $N_{\alpha}$.

From (12), a correction local fractional functional can be structured as

$$
u_{n+1}(t)=u_{n}(t)+{ }_{0} I_{t}^{(\alpha)}\left\{\xi^{\alpha}\left[L_{\alpha} u_{n}(s)+N_{\alpha} u_{n}(s)\right]\right\},
$$

where $\xi^{\alpha}$ denotes a fractal Lagrange multiplier; that is, $\delta^{\alpha} \widetilde{u}_{n}=$ $0[26]$.

Making use of (18), the new iteration formula reads as

$$
\begin{aligned}
\delta^{\alpha} u_{n+1}(t)= & \delta^{\alpha} u_{n}(t)+\delta^{\alpha}{ }_{0} I_{t}^{(\alpha)} \\
& \times\left\{\xi^{\alpha}\left[L_{\alpha} u_{n}(s)+N_{\alpha} \widetilde{u}_{n}(s)\right]\right\},
\end{aligned}
$$

which leads to

$$
\left(\xi^{\alpha}(\tau)\right)^{(\alpha)}=0, \quad 1+\left.\xi^{\alpha}(\tau)\right|_{\tau=t}=0
$$

where $\delta^{\alpha} \widetilde{u}_{n}$ denotes a restricted local fractional variation; that is, $\delta^{\alpha} \tilde{u}_{n}=0[26]$. 
Therefore, from (15) the fractal Lagrange multiplier can be identified as

$$
\xi^{\alpha}(\tau)=-1
$$

Submitting (17) into (13), we have that

$$
u_{n+1}(t)=u_{n}(t)+{ }_{0} I_{t}^{(\alpha)}\left\{-\left[L_{\alpha} u_{n}(s)+N_{\alpha} u_{n}(s)\right]\right\} .
$$

Consequently, the nondifferentiable solution can be written as

$$
u=\lim _{n \rightarrow \infty} u_{n} .
$$

For more results, see [31-36].

\section{Approximate Solutions}

Example 3. Consider sample local fractional linear transport equations arising in fractal porous media in the form

$$
\frac{\partial^{\alpha} u(x, t)}{\partial t^{\alpha}}+\frac{\partial^{\alpha} u(x, t)}{\partial x^{\alpha}}=0,
$$

with the initial condition

$$
u(x, 0)=\frac{x^{\alpha}}{\Gamma(1+\alpha)} .
$$

From (17) we derive the following iterative formula:

$$
\begin{aligned}
& u_{n+1}(x, t) \\
& \quad=u_{n}(x, t)+{ }_{0} I_{t}^{(\alpha)}\left\{-\left[\frac{\partial^{\alpha} u_{n}(x, s)}{\partial s^{\alpha}}+\frac{\partial^{\alpha} u_{n}(x, s)}{\partial x^{\alpha}}\right]\right\},
\end{aligned}
$$

where the initial value condition is

$$
u_{0}(x, t)=u(x, 0)=\frac{x^{\alpha}}{\Gamma(1+\alpha)} .
$$

From (21) the first approximate term reads as

$$
\begin{aligned}
u_{1}(x, t) & =u_{0}(x, t)+{ }_{0} I_{t}^{(\alpha)}\left\{-\left[\frac{\partial^{\alpha} u_{0}(x, s)}{\partial s^{\alpha}}+\frac{\partial^{\alpha} u_{0}(x, s)}{\partial x^{\alpha}}\right]\right\} \\
& =\frac{x^{\alpha}}{\Gamma(1+\alpha)}+{ }_{0} I_{t}^{(\alpha)}\left\{-\left[\frac{\partial^{\alpha} u_{0}(x, s)}{\partial s^{\alpha}}+\frac{\partial^{\alpha} u_{0}(x, s)}{\partial x^{\alpha}}\right]\right\} \\
& =\frac{x^{\alpha}}{\Gamma(1+\alpha)}-\frac{t^{\alpha}}{\Gamma(1+\alpha)} .
\end{aligned}
$$

In a like manner, the second approximate term is

$$
\begin{aligned}
u_{2}(x, t)= & u_{1}(x, t)+{ }_{0} I_{t}^{(\alpha)}\left\{-\left[\frac{\partial^{\alpha} u_{1}(x, s)}{\partial s^{\alpha}}+\frac{\partial^{\alpha} u_{1}(x, s)}{\partial x^{\alpha}}\right]\right\} \\
= & \frac{x^{\alpha}}{\Gamma(1+\alpha)}-\frac{t^{\alpha}}{\Gamma(1+\alpha)} \\
& +{ }_{0} I_{t}^{(\alpha)}\left\{-\left[\frac{\partial^{\alpha} u_{1}(x, s)}{\partial s^{\alpha}}+\frac{\partial^{\alpha} u_{1}(x, s)}{\partial x^{\alpha}}\right]\right\} \\
= & \frac{x^{\alpha}}{\Gamma(1+\alpha)}-\frac{t^{\alpha}}{\Gamma(1+\alpha)} .
\end{aligned}
$$

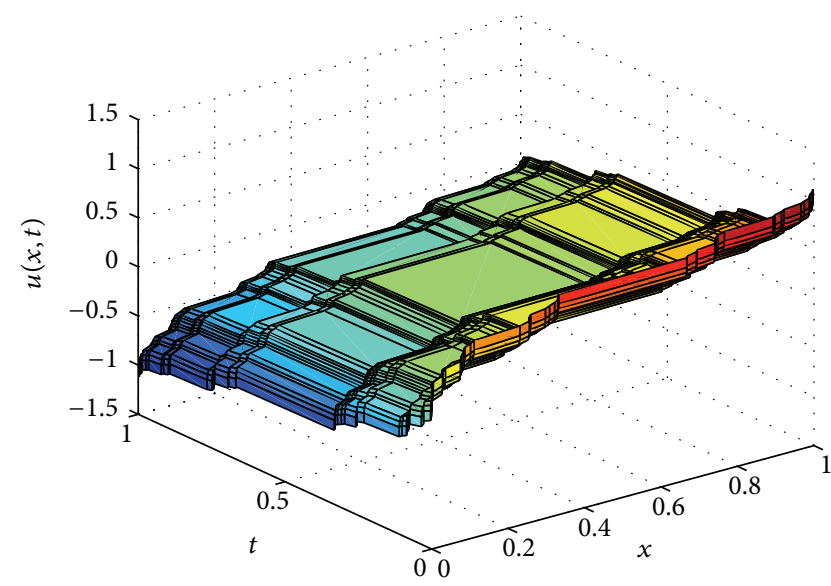

Figure 1: The plot of solution of (19) when $\alpha=\ln 2 / \ln 3$.

The third approximate term can be written as

$$
\begin{aligned}
u_{3}(x, t)= & u_{2}(x, t)+{ }_{0} I_{t}^{(\alpha)}\left\{-\left[\frac{\partial^{\alpha} u_{2}(x, s)}{\partial s^{\alpha}}+\frac{\partial^{\alpha} u_{2}(x, s)}{\partial x^{\alpha}}\right]\right\} \\
= & \frac{x^{\alpha}}{\Gamma(1+\alpha)}-\frac{t^{\alpha}}{\Gamma(1+\alpha)} \\
& +{ }_{0} I_{t}^{(\alpha)}\left\{-\left[\frac{\partial^{\alpha} u_{2}(x, s)}{\partial s^{\alpha}}+\frac{\partial^{\alpha} u_{2}(x, s)}{\partial x^{\alpha}}\right]\right\} \\
= & \frac{x^{\alpha}}{\Gamma(1+\alpha)}-\frac{t^{\alpha}}{\Gamma(1+\alpha)} .
\end{aligned}
$$

Continuing to calculate them in this manner, for $n>1$, we have that

$$
u_{n+1}(x, t)=u_{n}(x, t) .
$$

Hence, we obtain the nondifferentiable solution given by

$$
u(x, t)=\frac{x^{\alpha}}{\Gamma(1+\alpha)}-\frac{t^{\alpha}}{\Gamma(1+\alpha)},
$$

and its graph is shown in Figure 1.

Example 4. The sample local fractional linear transport equations arising in fractal porous media take the form

$$
\frac{\partial^{\alpha} u(x, t)}{\partial t^{\alpha}}+\frac{\partial^{\alpha} u(x, t)}{\partial x^{\alpha}}=0,
$$

subject to the initial condition

$$
u(x, 0)=E_{\alpha}\left(x^{\alpha}\right) .
$$

In view of (17) we structure the following iterative formula:

$$
\begin{aligned}
& u_{n+1}(x, t) \\
& \quad=u_{n}(x, t)+{ }_{0} I_{t}^{(\alpha)}\left\{-\left[\frac{\partial^{\alpha} u_{n}(x, s)}{\partial s^{\alpha}}+\frac{\partial^{\alpha} u_{n}(x, s)}{\partial x^{\alpha}}\right]\right\},
\end{aligned}
$$


where the initial value condition is expressed by

$$
u_{0}(x, t)=u(x, 0)=E_{\alpha}\left(x^{\alpha}\right) .
$$

In view of (31), we give the first approximation given by

$$
\begin{aligned}
u_{1}(x, t) & =u_{0}(x, t)+{ }_{0} I_{t}^{(\alpha)}\left\{-\left[\frac{\partial^{\alpha} u_{0}(x, s)}{\partial s^{\alpha}}+\frac{\partial^{\alpha} u_{0}(x, s)}{\partial x^{\alpha}}\right]\right\} \\
& =E_{\alpha}\left(x^{\alpha}\right)\left[1-\frac{t^{\alpha}}{\Gamma(1+\alpha)}\right] .
\end{aligned}
$$

Similarly, we obtain that

$$
\begin{aligned}
& u_{2}(x, t)=u_{1}(x, t)+{ }_{0} I_{t}^{(\alpha)}\left\{-\left[\frac{\partial^{\alpha} u_{1}(x, s)}{\partial s^{\alpha}}+\frac{\partial^{\alpha} u_{1}(x, s)}{\partial x^{\alpha}}\right]\right\} \\
& =E_{\alpha}\left(x^{\alpha}\right)\left[1-\frac{t^{\alpha}}{\Gamma(1+\alpha)}\right] \\
& +{ }_{0} I_{t}^{(\alpha)}\left\{-\left[\frac{\partial^{\alpha} u_{1}(x, s)}{\partial s^{\alpha}}+\frac{\partial^{\alpha} u_{1}(x, s)}{\partial x^{\alpha}}\right]\right\} \\
& =E_{\alpha}\left(x^{\alpha}\right)\left[1-\frac{t^{\alpha}}{\Gamma(1+\alpha)}+\frac{t^{2 \alpha}}{\Gamma(1+2 \alpha)}\right] \text {, } \\
& u_{3}(x, t)=u_{2}(x, t)+{ }_{0} I_{t}^{(\alpha)}\left\{-\left[\frac{\partial^{\alpha} u_{2}(x, s)}{\partial s^{\alpha}}+\frac{\partial^{\alpha} u_{2}(x, s)}{\partial x^{\alpha}}\right]\right\} \\
& =E_{\alpha}\left(x^{\alpha}\right)\left[1-\frac{t^{\alpha}}{\Gamma(1+\alpha)}+\frac{t^{2 \alpha}}{\Gamma(1+2 \alpha)}\right] \\
& +{ }_{0} I_{t}^{(\alpha)}\left\{-\left[\frac{\partial^{\alpha} u_{2}(x, s)}{\partial s^{\alpha}}+\frac{\partial^{\alpha} u_{2}(x, s)}{\partial x^{\alpha}}\right]\right\} \\
& =E_{\alpha}\left(x^{\alpha}\right)\left[1-\frac{t^{\alpha}}{\Gamma(1+\alpha)}+\frac{t^{2 \alpha}}{\Gamma(1+2 \alpha)}-\frac{t^{3 \alpha}}{\Gamma(1+3 \alpha)}\right] \text {, } \\
& u_{4}(x, t)=u_{3}(x, t)+{ }_{0} I_{t}^{(\alpha)}\left\{-\left[\frac{\partial^{\alpha} u_{3}(x, s)}{\partial s^{\alpha}}+\frac{\partial^{\alpha} u_{3}(x, s)}{\partial x^{\alpha}}\right]\right\} \\
& =E_{\alpha}\left(x^{\alpha}\right)-E_{\alpha}\left(x^{\alpha}\right) \frac{t^{\alpha}}{\Gamma(1+\alpha)} \\
& +{ }_{0} I_{t}^{(\alpha)}\left\{-\left[\frac{\partial^{\alpha} u_{3}(x, s)}{\partial s^{\alpha}}+\frac{\partial^{\alpha} u_{3}(x, s)}{\partial x^{\alpha}}\right]\right\} \\
& =E_{\alpha}\left(x^{\alpha}\right)\left[1-\frac{t^{\alpha}}{\Gamma(1+\alpha)}+\frac{t^{2 \alpha}}{\Gamma(1+2 \alpha)}\right. \\
& \left.-\frac{t^{3 \alpha}}{\Gamma(1+3 \alpha)}+\frac{t^{4 \alpha}}{\Gamma(1+4 \alpha)}\right]
\end{aligned}
$$

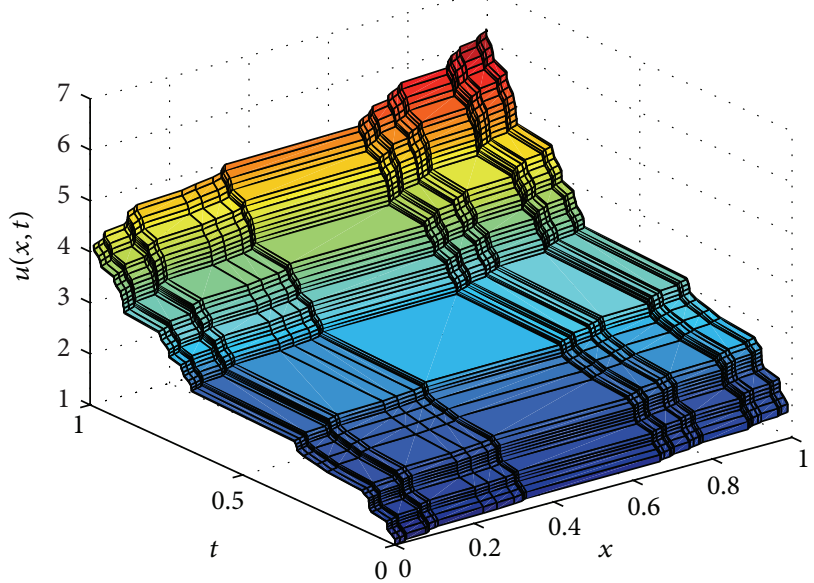

Figure 2: The plot of solution of (28) when $\alpha=\ln 2 / \ln 3$.

Hence, the nondifferentiable solution is given by

$$
\begin{aligned}
& u(x, t) \\
& \quad=\lim _{n \rightarrow \infty} u_{n}(x, t) \\
& \quad=\lim _{n \rightarrow \infty} E_{\alpha}\left(x^{\alpha}\right)\left[\sum_{k=0}^{\infty} \frac{t^{2 \alpha k}}{\Gamma(1+2 \alpha k)}-\sum_{k=0}^{\infty} \frac{t^{(2 k+1) \alpha}}{\Gamma[1+(2 k+1) \alpha]}\right] \\
& \quad=E_{\alpha}\left(x^{\alpha}\right)\left[\cosh _{\alpha}\left(t^{\alpha}\right)-\sinh _{\alpha}\left(t^{\alpha}\right)\right] .
\end{aligned}
$$

together with the graph shown in Figure 2.

Example 5. We now focus on the following local fractional linear transport equations arising in fractal porous media:

$$
\frac{\partial^{\alpha} u(x, t)}{\partial t^{\alpha}}+\frac{\partial^{\alpha} u(x, t)}{\partial x^{\alpha}}=0,
$$

subject to the initial condition

$$
u(x, 0)=\cos _{\alpha}\left(x^{\alpha}\right)
$$

In view of (17) the following iterative formula is given by

$$
\begin{aligned}
u_{n+1}(x, t)= & u_{n}(x, t) \\
& +{ }_{0} I_{t}^{(\alpha)}\left\{-\left[\frac{\partial^{\alpha} u_{n}(x, s)}{\partial s^{\alpha}}+\frac{\partial^{\alpha} u_{n}(x, s)}{\partial x^{\alpha}}\right]\right\},
\end{aligned}
$$

where the initial value condition is expressed by

$$
u_{0}(x, t)=u(x, 0)=\cos _{\alpha}\left(x^{\alpha}\right)
$$

and so on. 
Using (37) gives the approximate terms

$$
\begin{aligned}
& u_{1}(x, t)=u_{0}(x, t)+{ }_{0} I_{t}^{(\alpha)}\left\{-\left[\frac{\partial^{\alpha} u_{0}(x, s)}{\partial s^{\alpha}}+\frac{\partial^{\alpha} u_{0}(x, s)}{\partial x^{\alpha}}\right]\right\} \\
& =\cos _{\alpha}\left(x^{\alpha}\right)+{ }_{0} I_{t}^{(\alpha)}\left\{-\left[\frac{\partial^{\alpha} u_{0}(x, s)}{\partial s^{\alpha}}+\frac{\partial^{\alpha} u_{0}(x, s)}{\partial x^{\alpha}}\right]\right\} \\
& =\cos _{\alpha}\left(x^{\alpha}\right)+\sin _{\alpha}\left(x^{\alpha}\right) \frac{t^{\alpha}}{\Gamma(1+\alpha)}, \\
& u_{2}(x, t)=u_{1}(x, t)+{ }_{0} I_{t}^{(\alpha)}\left\{-\left[\frac{\partial^{\alpha} u_{1}(x, s)}{\partial s^{\alpha}}+\frac{\partial^{\alpha} u_{1}(x, s)}{\partial x^{\alpha}}\right]\right\} \\
& =\cos _{\alpha}\left(x^{\alpha}\right)+\sin _{\alpha}\left(x^{\alpha}\right) \frac{t^{\alpha}}{\Gamma(1+\alpha)} \\
& +{ }_{0} I_{t}^{(\alpha)}\left\{-\left[\frac{\partial^{\alpha} u_{1}(x, s)}{\partial s^{\alpha}}+\frac{\partial^{\alpha} u_{1}(x, s)}{\partial x^{\alpha}}\right]\right\} \\
& =\cos _{\alpha}\left(x^{\alpha}\right)+\sin _{\alpha}\left(x^{\alpha}\right) \frac{t^{\alpha}}{\Gamma(1+\alpha)} \\
& -\cos _{\alpha}\left(x^{\alpha}\right) \frac{t^{2 \alpha}}{\Gamma(1+2 \alpha)}, \\
& u_{3}(x, t)=u_{2}(x, t)+{ }_{0} I_{t}^{(\alpha)}\left\{-\left[\frac{\partial^{\alpha} u_{2}(x, s)}{\partial s^{\alpha}}+\frac{\partial^{\alpha} u_{2}(x, s)}{\partial x^{\alpha}}\right]\right\} \\
& =\cos _{\alpha}\left(x^{\alpha}\right)+\sin _{\alpha}\left(x^{\alpha}\right) \frac{t^{\alpha}}{\Gamma(1+\alpha)} \\
& -\cos _{\alpha}\left(x^{\alpha}\right) \frac{t^{2 \alpha}}{\Gamma(1+2 \alpha)} \\
& +{ }_{0} I_{t}^{(\alpha)}\left\{-\left[\frac{\partial^{\alpha} u_{2}(x, s)}{\partial s^{\alpha}}+\frac{\partial^{\alpha} u_{2}(x, s)}{\partial x^{\alpha}}\right]\right\} \\
& =\sin _{\alpha}\left(x^{\alpha}\right) \sum_{k=0}^{1}(-1)^{k} \frac{t^{(2 k+1) \alpha}}{\Gamma(1+(2 k+1) \alpha)} \\
& +\cos _{\alpha}\left(x^{\alpha}\right) \sum_{k=0}^{1}(-1)^{k} \frac{t^{2 k \alpha}}{\Gamma(1+2 k \alpha)} \\
& u_{4}(x, t)=u_{3}(x, t)+{ }_{0} I_{t}^{(\alpha)}\left\{-\left[\frac{\partial^{\alpha} u_{3}(x, s)}{\partial s^{\alpha}}+\frac{\partial^{\alpha} u_{3}(x, s)}{\partial x^{\alpha}}\right]\right\} \\
& =\sin _{\alpha}\left(x^{\alpha}\right) \sum_{k=0}^{1}(-1)^{k} \frac{t^{(2 k+1) \alpha}}{\Gamma(1+(2 k+1) \alpha)} \\
& +\cos _{\alpha}\left(x^{\alpha}\right) \sum_{k=0}^{1}(-1)^{k} \frac{t^{2 k \alpha}}{\Gamma(1+2 k \alpha)} \\
& +{ }_{0} I_{t}^{(\alpha)}\left\{-\left[\frac{\partial^{\alpha} u_{3}(x, s)}{\partial s^{\alpha}}+\frac{\partial^{\alpha} u_{3}(x, s)}{\partial x^{\alpha}}\right]\right\}
\end{aligned}
$$

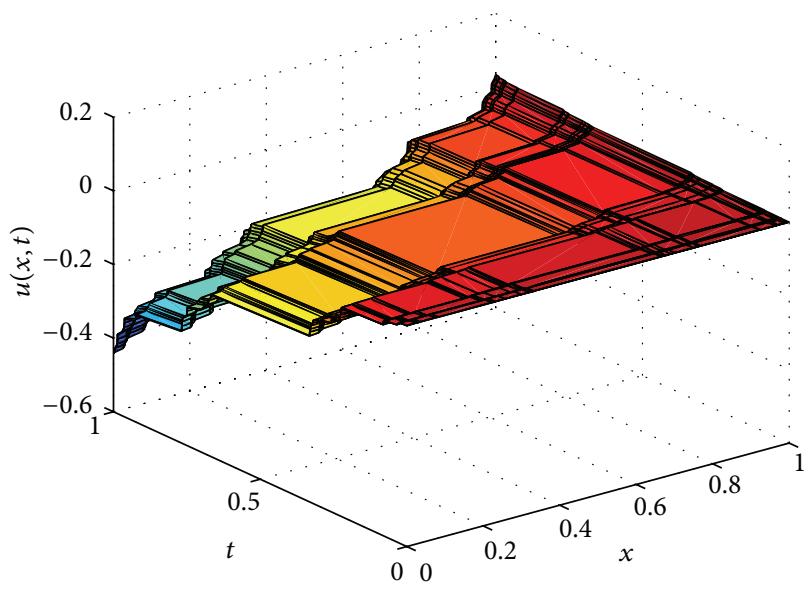

Figure 3: The plot of solution of (35) when $\alpha=\ln 2 / \ln 3$.

$$
\begin{aligned}
& =\sin _{\alpha}\left(x^{\alpha}\right) \sum_{k=0}^{2}(-1)^{k} \frac{t^{(2 k+1) \alpha}}{\Gamma(1+(2 k+1) \alpha)} \\
& +\cos _{\alpha}\left(x^{\alpha}\right) \sum_{k=0}^{2}(-1)^{k} \frac{t^{2 k \alpha}}{\Gamma(1+2 k \alpha)},
\end{aligned}
$$

and so on.

Therefore, the nondifferentiable solution of (35) can be written as

$$
\begin{aligned}
& u(x, t)= \lim _{n \rightarrow \infty} u_{n}(x, t) \\
&=\lim _{n \rightarrow \infty}\left[\sin _{\alpha}\left(x^{\alpha}\right) \sum_{k=0}^{\infty}(-1)^{k} \frac{t^{(2 k+1) \alpha}}{\Gamma(1+(2 k+1) \alpha)}\right. \\
&\left.\quad+\cos _{\alpha}\left(x^{\alpha}\right) \sum_{k=0}^{\infty}(-1)^{k} \frac{t^{2 k \alpha}}{\Gamma(1+2 k \alpha)}\right] \\
&=\lim _{n \rightarrow \infty}\left[\sin _{\alpha}\left(x^{\alpha}\right) \sin _{\alpha}\left(t^{\alpha}\right)+\cos _{\alpha}\left(x^{\alpha}\right) \cos _{\alpha}\left(t^{\alpha}\right)\right]
\end{aligned}
$$

and its graph is given in Figure 3.

Example 6. We discuss the following local fractional linear transport equations arising in fractal porous media:

$$
\frac{\partial^{\alpha} u(x, t)}{\partial t^{\alpha}}+\frac{\partial^{\alpha} u(x, t)}{\partial x^{\alpha}}=0,
$$

subject to the initial condition

$$
u(x, 0)=\sin _{\alpha}\left(x^{\alpha}\right) .
$$

From (17) the following iterative formula can be written as

$$
\begin{aligned}
u_{n+1}(x, t)= & u_{n}(x, t) \\
& +{ }_{0} I_{t}^{(\alpha)}\left\{-\left[\frac{\partial^{\alpha} u_{n}(x, s)}{\partial s^{\alpha}}+\frac{\partial^{\alpha} u_{n}(x, s)}{\partial x^{\alpha}}\right]\right\},
\end{aligned}
$$


where the initial value condition is given by

$$
u_{0}(x, t)=u(x, 0)=\sin _{\alpha}\left(x^{\alpha}\right)
$$

Making use of (43), we obtain the approximate terms

$$
\begin{aligned}
u_{1}(x, t)= & u_{0}(x, t)+{ }_{0} I_{t}^{(\alpha)}\left\{-\left[\frac{\partial^{\alpha} u_{0}(x, s)}{\partial s^{\alpha}}+\frac{\partial^{\alpha} u_{0}(x, s)}{\partial x^{\alpha}}\right]\right\} \\
= & \sin _{\alpha}\left(x^{\alpha}\right)+{ }_{0} I_{t}^{(\alpha)}\left\{-\left[\frac{\partial^{\alpha} u_{0}(x, s)}{\partial s^{\alpha}}+\frac{\partial^{\alpha} u_{0}(x, s)}{\partial x^{\alpha}}\right]\right\} \\
= & \sin _{\alpha}\left(x^{\alpha}\right)-\cos _{\alpha}\left(x^{\alpha}\right) \frac{t^{\alpha}}{\Gamma(1+\alpha)}, \\
u_{2}(x, t)= & u_{1}(x, t)+{ }_{0} I_{t}^{(\alpha)}\left\{-\left[\frac{\partial^{\alpha} u_{1}(x, s)}{\partial s^{\alpha}}+\frac{\partial^{\alpha} u_{1}(x, s)}{\partial x^{\alpha}}\right]\right\} \\
= & \sin _{\alpha}\left(x^{\alpha}\right)-\cos _{\alpha}\left(x^{\alpha}\right) \frac{t^{\alpha}}{\Gamma(1+\alpha)} \\
& +{ }_{0} I_{t}^{(\alpha)}\left\{-\left[\frac{\partial^{\alpha} u_{1}(x, s)}{\partial s^{\alpha}}+\frac{\partial^{\alpha} u_{1}(x, s)}{\partial x^{\alpha}}\right]\right\} \\
= & \sin _{\alpha}\left(x^{\alpha}\right)-\cos _{\alpha}\left(x^{\alpha}\right) \frac{t^{\alpha}}{\Gamma(1+\alpha)} \\
& -\sin _{\alpha}\left(x^{\alpha}\right) \frac{t^{2 \alpha}}{\Gamma(1+2 \alpha)},
\end{aligned}
$$$$
u_{3}(x, t)=u_{2}(x, t)+{ }_{0} I_{t}^{(\alpha)}\left\{-\left[\frac{\partial^{\alpha} u_{2}(x, s)}{\partial s^{\alpha}}+\frac{\partial^{\alpha} u_{2}(x, s)}{\partial x^{\alpha}}\right]\right\}
$$$$
=\sin _{\alpha}\left(x^{\alpha}\right)-\cos _{\alpha}\left(x^{\alpha}\right) \frac{t^{\alpha}}{\Gamma(1+\alpha)}
$$$$
-\sin _{\alpha}\left(x^{\alpha}\right) \frac{t^{2 \alpha}}{\Gamma(1+2 \alpha)}
$$$$
+{ }_{0} I_{t}^{(\alpha)}\left\{-\left[\frac{\partial^{\alpha} u_{2}(x, s)}{\partial s^{\alpha}}+\frac{\partial^{\alpha} u_{2}(x, s)}{\partial x^{\alpha}}\right]\right\}
$$$$
=\sin _{\alpha}\left(x^{\alpha}\right)-\cos _{\alpha}\left(x^{\alpha}\right) \frac{t^{\alpha}}{\Gamma(1+\alpha)}
$$$$
-\sin _{\alpha}\left(x^{\alpha}\right) \frac{t^{2 \alpha}}{\Gamma(1+2 \alpha)}+\cos _{\alpha}\left(x^{\alpha}\right) \frac{t^{3 \alpha}}{\Gamma(1+3 \alpha)},
$$$$
u_{4}(x, t)=u_{3}(x, t)+{ }_{0} I_{t}^{(\alpha)}\left\{-\left[\frac{\partial^{\alpha} u_{3}(x, s)}{\partial s^{\alpha}}+\frac{\partial^{\alpha} u_{3}(x, s)}{\partial x^{\alpha}}\right]\right\}
$$$$
=\sin _{\alpha}\left(x^{\alpha}\right)-\cos _{\alpha}\left(x^{\alpha}\right) \frac{t^{\alpha}}{\Gamma(1+\alpha)}
$$$$
-\sin _{\alpha}\left(x^{\alpha}\right) \frac{t^{2 \alpha}}{\Gamma(1+2 \alpha)}+\cos _{\alpha}\left(x^{\alpha}\right) \frac{t^{3 \alpha}}{\Gamma(1+3 \alpha)}
$$$$
+{ }_{0} I_{t}^{(\alpha)}\left\{-\left[\frac{\partial^{\alpha} u_{3}(x, s)}{\partial s^{\alpha}}+\frac{\partial^{\alpha} u_{3}(x, s)}{\partial x^{\alpha}}\right]\right\}
$$

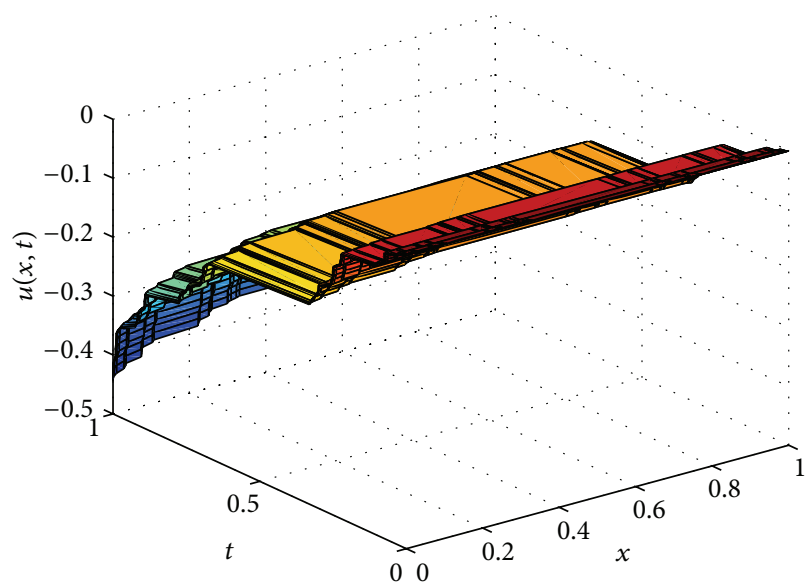

FIGURE 4: The plot of solution of (41) when $\alpha=\ln 2 / \ln 3$.

$$
\begin{aligned}
= & \sin _{\alpha}\left(x^{\alpha}\right) \sum_{k=0}^{2}(-1)^{k} \frac{t^{2 k \alpha}}{\Gamma(1+2 k \alpha)} \\
& -\cos _{\alpha}\left(x^{\alpha}\right) \sum_{k=0}^{1}(-1)^{k} \frac{t^{(2 k+1) \alpha}}{\Gamma(1+(2 k+1) \alpha)},
\end{aligned}
$$$$
\vdots
$$

and so on.

Therefore, the nondifferentiable solution of (41) reads as

$$
\begin{aligned}
& u(x, t)= \lim _{n \rightarrow \infty} u_{n}(x, t) \\
&=\lim _{n \rightarrow \infty}\left[\sin _{\alpha}\left(x^{\alpha}\right) \sum_{k=0}^{\infty}(-1)^{k} \frac{t^{2 k \alpha}}{\Gamma(1+2 k \alpha)}\right. \\
&\left.\quad-\cos _{\alpha}\left(x^{\alpha}\right) \sum_{k=0}^{\infty}(-1)^{k} \frac{t^{(2 k+1) \alpha}}{\Gamma(1+(2 k+1) \alpha)}\right] \\
&=\sin _{\alpha}\left(x^{\alpha}\right) \cos _{\alpha}\left(t^{\alpha}\right)-\cos _{\alpha}\left(x^{\alpha}\right) \sin _{\alpha}\left(t^{\alpha}\right)
\end{aligned}
$$

and its graph is given in Figure 4.

\section{Conclusions}

Local fractional calculus theory is a tool for modeling the nondifferentiable problems for science and engineering. In this work we studied the local fractional linear transport equations arising in fractal porous media by using the local fractional variational iteration method. The solutions with nondifferentiable functions were also obtained and some examples were also discussed. These results show the reliabilities and efficiencies of the proposed local fractional variational iteration method. 


\section{Conflict of Interests}

The authors declare that there is no conflict of interests regarding the publication of this paper.

\section{Acknowledgments}

The work was supported by the National Natural Science Foundation of China under Grant no. 71173060. It was also supported by the China Postdoctoral Science Foundation under Grant no. 2013M541351.

\section{References}

[1] V. Agoshkov, Boundary Value Problems for Transport Equations, Modeling and Simulation in Science, Engineering and Technology, Birkhäuser, Boston, Mass, USA, 1998.

[2] L. Dumas and F. Golse, "Homogenization of transport equations," SIAM Journal on Applied Mathematics, vol. 60, no. 4, pp. 1447-1470, 2000.

[3] E. Weinan, "Homogenization of linear and nonlinear transport equations," Communications on Pure and Applied Mathematics, vol. 45, no. 3, pp. 301-326, 1992.

[4] R. W. Schunk, "Transport equations for aeronomy," Planetary and Space Science, vol. 23, no. 3, pp. 437-485, 1975.

[5] K. Blotekjaer, "Transport equations for electrons in two-valley semiconductors," IEEE Transactions on Electron Devices, vol. 17, no. 1 , pp. 38-47, 1970.

[6] O. Betbeder-Matibet and P. Nozieres, "Transport equations in clean superconductors," Annals of Physics, vol. 51, no. 3, pp. 392417, 1969.

[7] B. J. Daly and F. H. Harlow, "Transport equations in turbulence," Physics of Fluids, vol. 13, no. 11, pp. 2634-2649, 1970.

[8] H.-T. Elze, M. Gyulassy, and D. Vasak, "Transport equations for the QCD quark Wigner operator," Nuclear Physics B, vol. 276, no. 3-4, pp. 706-728, 1986.

[9] A. B. Mikhailovskii and V. S. Tsypin, "Transport equations of plasma in a curvilinear magnetic field," Beiträge aus der Plasmaphysik, vol. 24, no. 4, pp. 335-354, 1984.

[10] B. S. Tanenbaum, "Transport equations for a gas mixture," Physics of Fluids, vol. 8, no. 4, pp. 683-686, 1965.

[11] B. Perthame, Transport Equations in Biology, Springer, Berlin, Germany, 2006.

[12] G. Petrova and B. Popov, "Linear transport equations with discontinuous coefficients," Communications in Partial Differential Equations, vol. 24, no. 9-10, pp. 1849-1873, 1999.

[13] D. B. Ingham and I. Pop, Transport Phenomena in Porous Media, vol. 3, Elsevier, Oxford, UK, 2005.

[14] H. E. Stanley, "Application of fractal concepts to polymer statistics and to anomalous transport in randomly porous media," Journal of Statistical Physics, vol. 36, no. 5-6, pp. 843860, 1984.

[15] M. Sahimi, "Fractal and superdiffusive transport and hydrodynamic dispersion in heterogeneous porous media," Transport in Porous Media, vol. 13, no. 1, pp. 3-40, 1993.

[16] S. P. Rigby and L. F. Gladden, "NMR and fractal modelling studies of transport in porous media," Chemical Engineering Science, vol. 51, no. 10, pp. 2263-2272, 1996.
[17] M. Colotin, G. O. Pompilian, P. Nica, S. Gurlui, V. Paun, and M. Agop, "Fractal transport phenomena through the scale relativity model," Acta Physica Polonica A, vol. 116, no. 2, pp. 157-164, 2009.

[18] R. Klages, G. Radons, and I. M. Sokolov, Anomalous Transport: Foundations and Applications, John Wiley \& Sons, New York, NY, USA, 2008.

[19] X. Leoncini, L. Kuznetsov, and G. M. Zaslavsky, "Evidence of fractional transport in point vortex flow," Chaos, Solitons \& Fractals, vol. 19, no. 2, pp. 259-273, 2004.

[20] G. M. Zaslavsky, "Chaos, fractional kinetics, and anomalous transport," Physics Report, vol. 371, no. 6, pp. 461-580, 2002.

[21] V. E. Tarasov, "Transport equations from Liouville equations for fractional systems," International Journal of Modern Physics B, vol. 20, no. 3, pp. 341-353, 2006.

[22] E. Lutz, "Fractional transport equations for Lévy stable processes," Physical Review Letters, vol. 86, no. 11, pp. 2208-2211, 2001.

[23] V. V. Uchaikin and R. T. Sibatov, "Fractional theory for transport in disordered semiconductors," Communications in Nonlinear Science and Numerical Simulation, vol. 13, no. 4, pp. 715-727, 2008.

[24] R. Metzler and J. Klafter, "The restaurant at the end of the random walk: recent developments in the description of anomalous transport by fractional dynamics," Journal of Physics A: Mathematical and General, vol. 37, no. 31, pp. R161-R208, 2004.

[25] A. Kadem, Y. Luchko, and D. Baleanu, "Spectral method for solution of the fractional transport equation," Reports on Mathematical Physics, vol. 66, no. 1, pp. 103-115, 2010.

[26] X.-J. Yang, Advanced Local Fractional Calculus and Its Applications, World Science Publisher, New York, NY, USA, 2012.

[27] X. J. Yang, Local Fractional Functional Analysis and Its Applications, Asian Academic Publisher, Hong Kong, 2011.

[28] X. J. Yang, D. Baleanu, and J. H. He, "Transport equations in fractal porous media within fractional complex transform method," Proceedings of the Romanian Academy A, vol. 14, no. 4, pp. 287-292, 2013.

[29] C.-G. Zhao, A.-M. Yang, H. Jafari, and A. Haghbin, "The YangLaplace transform for solving the IVPs with local fractional derivative," Abstract and Applied Analysis, vol. 2014, Article ID 386459, 5 pages, 2014.

[30] A.-M. Yang, Y.-Z. Zhang, C. Cattani et al., "Application of local fractional series expansion method to solve Klein-Gordon equations on Cantor sets," Abstract and Applied Analysis, vol. 2014, Article ID 372741, 6 pages, 2014.

[31] X. J. Yang and D. Baleanu, "Fractal heat conduction problem solved by local fractional variation iteration method," Thermal Science, vol. 17, no. 2, pp. 625-628, 2013.

[32] X. J. Yang and D. Baleanu, "Local fractional variational iteration method for Fokker-Planck equation on a Cantor set," Acta Universitaria, vol. 23, no. 2, pp. 3-8, 2013.

[33] A. M. Yang, Z. S. Chen, H. M. Srivastava, and X. J. Yang, "Application of the local fractional series expansion method and the variational iteration method to the Helmholtz equation involving local fractional derivative operators," Abstract and Applied Analysis, vol. 2013, Article ID 259125, 6 pages, 2013.

[34] D. Baleanu, J. A. Tenreiro Machado, C. Cattani, M. C. Baleanu, and X.-J. Yang, "Local fractional variational iteration and 
decomposition methods for wave equation on Cantor sets within local fractional operators," Abstract and Applied Analysis, vol. 2014, Article ID 535048, 6 pages, 2014.

[35] J. H. He, "Local fractional variational iteration method for fractal heat transfer in silk cocoon hierarchy," Nonlinear Science Letters A, vol. 4, no. 1, pp. 15-20, 2013.

[36] C. F. Liu, S. S. Kong, and S. J. Yuan, "Reconstructive schemes for variational iteration method within Yang-Laplace transform with application to fractal heat conduction problem," Thermal Science, vol. 17, no. 3, pp. 715-721, 2013. 


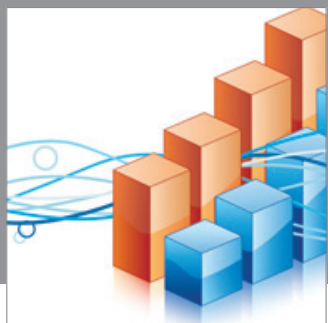

Advances in

Operations Research

mansans

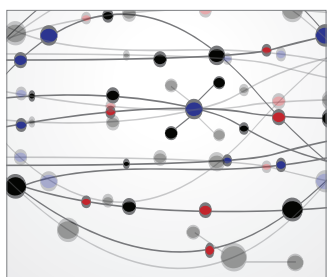

The Scientific World Journal
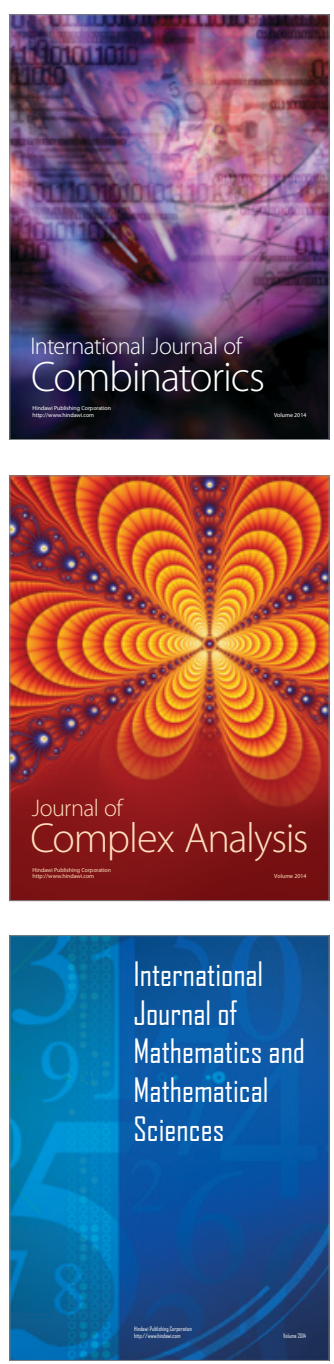
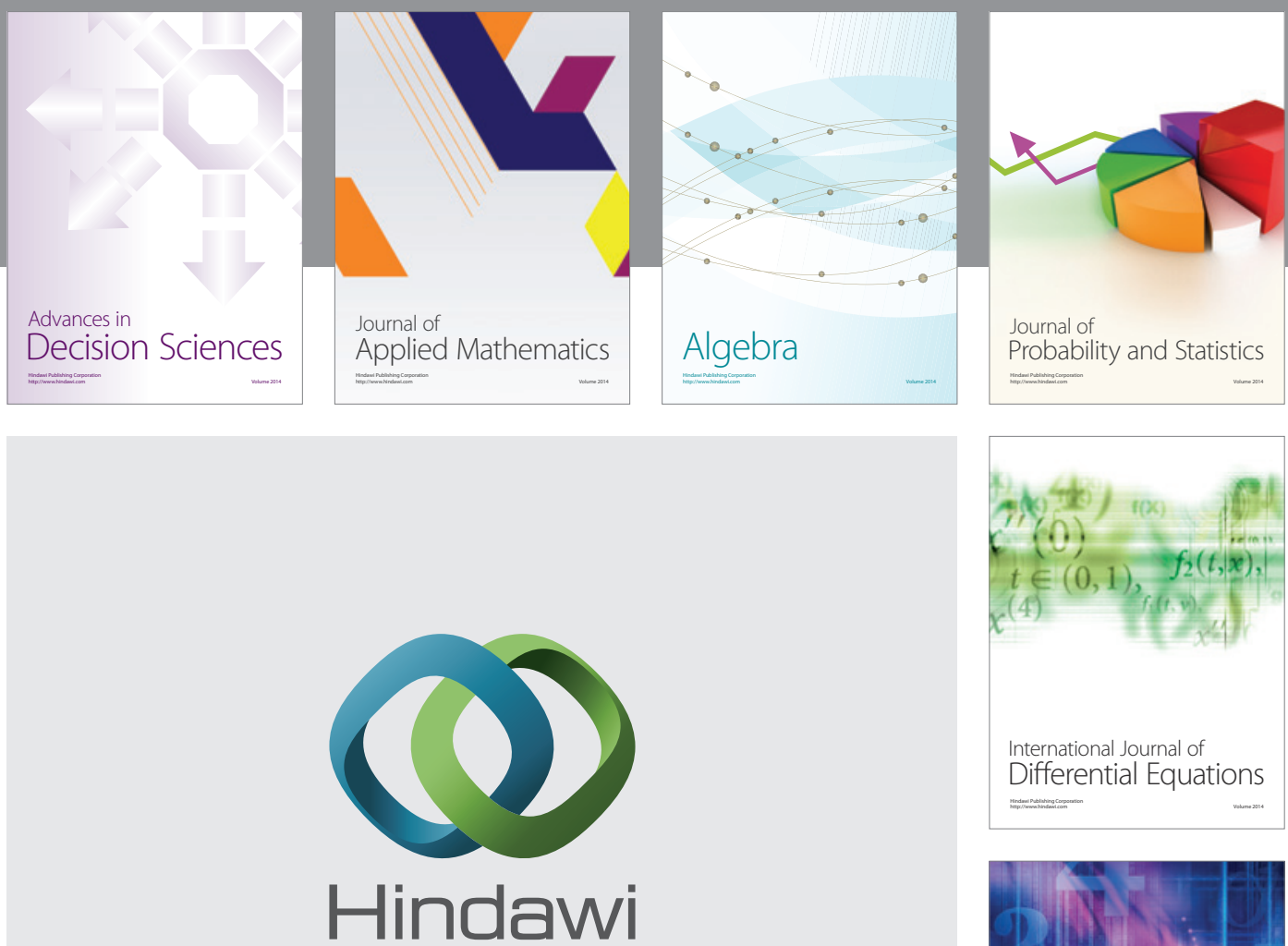

Submit your manuscripts at http://www.hindawi.com
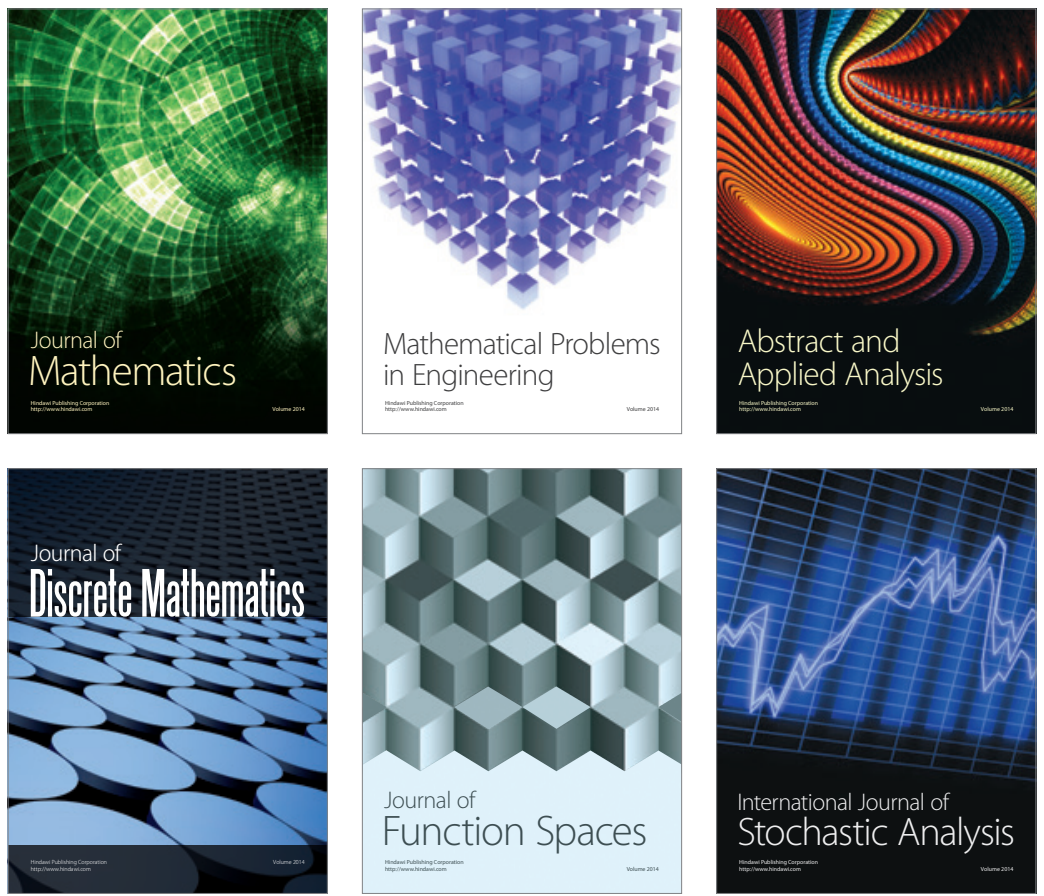

Journal of

Function Spaces

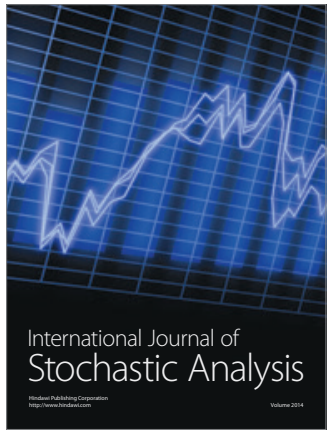

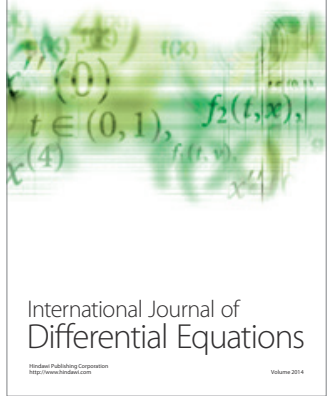
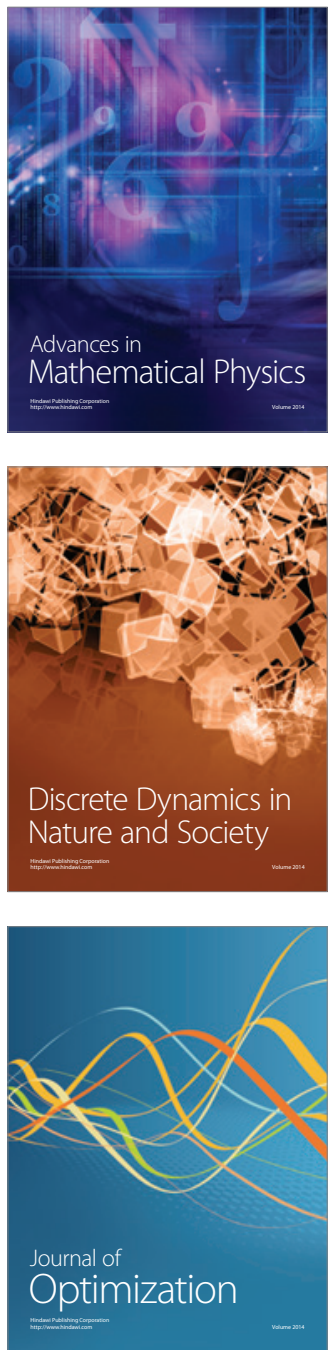